\title{
Status of anaerobic digestion pretreatment of high solid waste*
}

\author{
Wan-Qian Guo, Chang Huang and Zi-Zeng Yang \\ State Key Laboratory of Urban Water Resource and Environment, \\ Harbin Institute of Technology, \\ Harbin 150090, PR China. \\ 'E-mail: guowanqian@126.com
}

\begin{abstract}
Compared with low-solid state, high-solid anaerobic digestion has the advantages of requiring less reactor volume, less energy input for heating and mixing and producing nutrient-richer fertilizer. Besides, it also has a higher net energy yield. It is urgently necessary to pay attention to the resource of solid waste. However, it is considered that the hydrolysis stage is a rate-limited step in the anaerobic digestion. Therefore, a various pretreatments have been applied to improve the hydrolysis process. In this paper, three pretreatment methods which can be used in engineering were reviewed.
\end{abstract}

Keywords: High-solid; Anaerobic Digestion; Pretreatment; Thermal; NaOH; Ultrasonic.

\section{Introduction}

In recent years, with the development of China's economy, the level of urbanization and the improvement of people's living standards, the city's biomass waste showed a significant growth trend. The annual growth rate of solid waste from 2010 to 2014 was $3 \%$. In 2014, the quantity of municipal solid waste was 178.602 million tons[1].

Anaerobic digestion (AD) is a biological process in which organic matter is degraded and converted to clean biogas under anaerobic conditions. Traditional anaerobic digestion is normally carried out at low-solid state. When compared to low-solid anaerobic digestion, high-solid anaerobic digestion requires less reactor volume, requires less energy input for heating and mixing, produces nutrient-richer fertilizer, and has a higher net energy yield[2].

Hydrolysis is the rate-limiting step of the overall processes which release the intracellular and extracellular matters. Pretreatment have been studied to promote hydrolysis.

\footnotetext{
* This work was financially supported by young top-notch talent teachers by Harbin Institute of Technology (AUGA5710052514). The authors also gratefully acknowledge the financial support by State Key Laboratory of Urban Water Resource and Environment (2014TS06).
} 


\section{Thermal Pretreatment}

Thermal pretreatment has been suggested as an effective method by which to accelerate hydrolysis during conventional anaerobic digestion of low-solids sludge, and thereby shorten digestion time and enhance biogas production[3].

Applying thermal pretreatment to high-solid sludge anaerobic digestion is reasonable considering that conventional anaerobic digestion and high-solid sludge anaerobic digestion are based on the same fundamental biochemical processes. Previous research on thermal pretreatment of high-solid sludge anaerobic digestion divided into high temperatures $\left(120-180^{\circ} \mathrm{C}\right.$ ) and low temperatures $\left(50-90{ }^{\circ} \mathrm{C}\right)$ pretreatment. After thermal hydrolysis pretreatment, which reduces sludge viscosity and increases the fraction of soluble COD, highsolids anaerobic digestion is stable, produces highly stabilized biosolids, and greatly increases biogas generation[4].

Liao et al. [3] found that after low temperature thermal treatment, a large amount of organic matter in sludge solids was dissolved, and sludge flow ability was enhanced significantly. Xue et al. [5] found that both the low temperature thermal pretreatment and high temperature thermal pretreatment positively affected the solubilization of organics in sludge. In the biodegradability batch experiments, the low temperature thermal pretreatment did not improve the total gas volume, while the high temperature thermal pretreatment did. Zhu et al. [6] found that the oil extraction amount of kitchen waste after thermal hydrolysis pretreatment was increased significantly. the highest $(67.7 \mathrm{ml} / \mathrm{kg})$ was achieved under the conditions of $150{ }^{\circ} \mathrm{C}, 40 \%$ water added and $60 \mathrm{~min}$ thermal hydrolysis, which was 2. 65 times higher than the control group.

\section{NaOH Pretreatment}

Alkaline pretreatment can effectively dissolve the cellulose into dissolved organic carbon compounds, so it is easy to be used by microorganisms. The effect is related to the type and dosage of alkaline [7]. $\mathrm{NaOH}$ pretreatment is usually used in sewage sludge and straw anaerobic digestion.

Some researchers proposed that $\mathrm{NaOH}$ pretreatment can enhance sludge solubilisation and methane yields. Zhang et al. [8]thought the reason was mainly due to the increasing $\mathrm{pH}$ value. The increasing $\mathrm{pH}$ value changed cell osmotic pressure in dewatered activated sludge resulting in extracellular polymeric substances solubilisation and cell lysis. Cho et al. [9] found the solubilisation efficiency and methane yield of the sludge increased significantly. The SCOD, soluble protein and carbohydrate concentration increased with the increase of the $\mathrm{NaOH}$ dosage. During high solid anaerobic digestion process, although high 
$\mathrm{NaOH}$ does inhibited methanogenic activity, moderate $\mathrm{NaOH}$ does was able to improve cumulative methane yield[8].

The straw contains a high content of lignin, which cannot be well utilized by anaerobic bacteria in high solid anaerobic digestion process. $\mathrm{NaOH}$ pretreatment can destroy the complex structure of the lignin to enhance its high solid anaerobic digestion. Chen et al. [10] thought that not only lignocellulose but also some nitrogenous materials were destroyed or broken down by alkaline treatment. The lignin structure was destroyed through alkaline treatment that caused the decreased lignin, but the crystallinity index of cellulose increased. Pang et al. [11] investigated the effect of temperature and chemical pretreatment on anaerobic biogasification of corn stalk. The result showed that $\mathrm{NaOH}$ was the optimal reagent for pretreatment to increase the yield of biogas at $35^{\circ} \mathrm{C}$ by $77.0 \%$ than that of anaerobic digestion without pretreatment at the same temperature.

\section{Ultrasonic Pretreatment}

As a new pretreatment method, ultrasonic technology is mainly used in the anaerobic digestion of sludge. Meanwhile it is used in the anaerobic digestion of kitchen waste and cow-dung.

Ultrasound could destroy the sludge flocs and the cytoderm through the shear force caused by Cavitation bubble breakup, so that the EPS and intracellular substances, such as protein, nucleic acid, sugar and other substances release into the solution. As a consequence, the sludge anaerobic digestion improved and the value of COD increased. The main influence factors of ultrasonic pretreatment are ultrasonic time, power density and $\mathrm{pH}$ of sludge. Vlyssides et al. [12] studied the effect of ultrasonic pretreatment on the solubilization of sludge. The results showed that the increase of total solid solubility increased with the energy input increment.

Appropriate intensity of ultrasound can effectively improve the activity of the enzyme and promote cell growth. Some researchers studied the effect of ultrasound pretreatment on cow-dung anaerobic digestion. Results showed that the ultrasound pretreatment with proper intensity was benefited to cow-dung anaerobic digestion.

\section{Conclusion}

Pretreatment is an effect way to improve the performance of high solid anaerobic digestion. The main effect is to improve the solubilisation of the solid waste in the hydrolysis process. At the same time, some pretreatment methods can effectively improve the yield of gas and acid production. Taking into 
account the actual industrial applications, the study should focus on the relatively low energy consumption.

\section{References}

1. STATISTICAL YEARBOOK OF CHINA, (National Bureau of Statistics of the People's Republic of China), http://www.stats.gov.cn/tjsj/ndsj/

2. Daniel I. Massé \& Noori M. Cata Saady, Dry anaerobic digestion of high solids content dairy manure at high organic loading rates in psychrophilic sequence batch reactor, Appl Microbiol. Biotechnol. 99, 4521 (2015) .

3. Xiaocong Liao, Huan Li, et al, Accelerated high-solids anaerobic digestion of sewage sludge using low-temperature thermal pretreatment, Int. Biodeterior. Biodegrad. 106, 141 (2016)

4. Domenec Jolis,High-Solids Anaerobic Digestion of Municipal Sludge Pretreated by Thermal Hydrolysis, Water Environ. Res. 80, 654 (2008).

5. Yonggang Xue, Huajie Liu, et al, Effects of thermal hydrolysis on organic matter solubilization and anaerobic digestion of high solid sludge, Chem. Eng. J. 264, 174 (2015).

6. Jinlong Zhu, Zimin Wei, et al, Impacts of liquid substances transformation of kitchen waste with hydrothermal pretreatment, Research of Environmental Sciences, 28, 440 (2015).(In Chinese)

7. Yuanyuan Yan, Xiaoguang Liu, et al, Review on Pretreatment of Anaerobic Sludge Digestion, Sichuan Environment,31, 113 (2012). (In Chinese)

8. Shuting Zhang, et al, Influence of $\mathrm{NaOH}$ and thermal pretreatment on dewatered activated sludge solubilisation and subsequent anaerobic digestion: Focused on high-solid state, Bioresour.Technol.185, 171 (2015) .

9. Si-Kyung Cho, Hyun-Jun Ju, et al, Alkaline-mechanical pretreatment process for enhanced anaerobic digestion of thickened waste activated sludge with a novel crushing device: Performance evaluation and economic analysis, Bioresour. Technol. 165, 183 (2014) .

10. Guangyin Chen, Zheng Zheng, et al, Effect of alkaline treatment on anaerobic digestion of rice straw, Environmental Science. 31, 2208 (2010). (In Chinese)

11. Yunzhi Pang, Xiujin Luo, et al, Effect of temperature and chemical pretreatment on anaerobic biogasification of corn stalk, Chinese Journal of Bioprocess Engineering. 3, 37 (2005). (In Chinese)

12. A.G. Vlyssides, P.K. Karlis, Thermal-alkaline solubilization of waste activated sludge as a pre-treatmen stage for anaerobic digestion, Bioresour. Technol. 91, 201 (2004) . 